\title{
Comparative Proteomics of Human Milk From Eight Cities in China During Six Months of Lactation in the Chinese Human Milk Project Study
}

\begin{abstract}
Ratna Nurmalita Sari ${ }^{1+}$, Jiancun Pan ${ }^{2,3+}$, Wenyuan Zhang ${ }^{1+}$, Yuanyuan $\mathrm{Li}^{2,3}$, Huiquan Zhu ${ }^{1}$, Xiaoyang Pang ${ }^{1}$, Shuwen Zhang ${ }^{1}$, Shilong Jiang ${ }^{2,3 *}$, Jing $L u^{1,4 *}$ and Jiaping $L v^{1 *}$

${ }^{1}$ Institute of Food Science and Technology, Chinese Academy of Agricultural Science, Beijing, China, ${ }^{2}$ Nutrition and Metabolism Research Division, Innovation Center, Heilongjiang Feihe Dairy Co., Ltd., Beijing, China, ${ }^{3}$ PKUHSC-China Feihe Joint Research Institute of Nutrition and Healthy Lifespan Development, Beijing, China, ${ }^{4}$ School of Food and Health, Beijing Business and Technology University, Beijing, China
\end{abstract}

OPEN ACCESS

Edited by:

Aida Serra

IMDEA Food Institute, Spain

Reviewed by:

Ahmed A. Zaky,

National Research Centre, Egypt Antía González Pereira,

University of Vigo, Spain

*Correspondence:

Shilong Jiang

jiangshilong@feihe.com

Jing Lu

lujing@btbu.edu.cn

Jiaping LV

kjdairy@126.com

†These authors have contributed equally to this work

Specialty section:

This article was submitted to Food Chemistry,

a section of the journal

Frontiers in Nutrition

Received: 18 March 2021

Accepted: 14 June 2021

Published: 12 August 2021

Citation:

Sari RN, Pan J, Zhang W, Li Y, Zhu H, Pang $X$, Zhang $S$, Jiang $S$, Lu J and Lv J (2021) Comparative Proteomics of Human Milk From Eight Cities in China During Six Months of Lactation in the Chinese Human Milk Project

Study. Front. Nutr. 8:682429.

doi: 10.3389/fnut.2021.682429
Human milk $(\mathrm{HM})$ is the golden standard of infant nutrition that can protect immature body function and enhance nutrition metabolism to ensure infant growth. Region specificity and lactation period could change the protein composition in HM. In this research, proteomics analysis was used to compare proteomes across eight cities, namely Harbin, Lanzhou, Guangzhou, Chengdu, Jinhua, Weihai, Zhengzhou, and Beijing, which represented the northeast, northwest, southeast, southwest, east, and north and central regions of China,. Proteins varied significantly among the cities. These different proteins were mainly involved in the process of platelet degranulation, innate immune response, and triglyceride metabolic process, which might be due to different living environments. These differences also lead to variation in protection and fat metabolism from mothers to infants in different cities. Four proteins were expressed differently during 6 months of lactation, namely Dipeptidyl peptidase 1, Lysozyme C, Carbonic anhydrase 6, and Chordin-like protein 2. The changes in these proteins might be because of the change of growth needs of the infants. The findings from our results might help to improve the understanding of $\mathrm{HM}$ as well as to design infant formula.

Keywords: Chinese human milk, human milk, cities, lactation period, proteomics

\section{INTRODUCTION}

Human milk (HM) is a complex liquid that contains variative compositions among mothers. It consists of true solutions, colloids, membranes, membrane-bound globules, and cells (1). The exclusive breastfeeding period of the first 6 months then continued up to 2 years is considered as the ideal standard for infant feeding (2). During the exclusive breastfeeding period, HM acts as the single source of nutrients that supports the immature immunity and metabolism of the infant (3). Meanwhile, the composition of HM is altered by many factors such as maternal factors, lactation stages, environmental exposures, regions, ethnicities, handling, and storage $(1,4)$.

Human milk contains myriad proteins that play a role in the biological process of infants such as immunological, antimicrobial, and developmental functions $(5,6)$. Recently, proteomics has become a robust approach to explore the overall biological function of HM proteins (7). Previously, proteomes variation of $\mathrm{HM}$ serum and milk fat globule membrane of individual mothers and 
variation of HM and ruminant's milk have been observed (3, 8-10). Those prior studies found that HM proteome contributed to various functions including immune protection, biological growth, and maturation of the digestive tract. HM profile, including lipid, oligosaccharides, and distinct patterns of microbiota varied across the population $(11,12)$. However, not so many studies emphasized the variation of protein composition, especially low abundant ones in the different regions. The variation of quantitative proteomes between Chinese and Dutch HM serum and the different proteomes across Chinese ethnicity and geographic location has been revealed $(13,14)$. They studied milk serum proteome of four different regions in China, mainly focused on the western region of China, namely Yunnan, Gansu, Xinjiang, and Inner Mongolia. To our knowledge, the milk proteins, especially low abundant proteins were not wellinvestigated in the eastern region of China.

Thus, HM proteomes in eight cities of China, which represented northeast, northwest, southeast, southwest, east, north, and central China, during the first 6 months of lactation were investigated in the present study by using proteomics methods with a bigger number of samples than prior studies. The result of this research will build in-depth understanding of proteomes in each city for further use, for instance, the development of infant formula for the infants that could not access sufficient HM.

\section{MATERIALS AND METHODS}

\section{Materials}

All the reagents were provided in analytical grade and suitable for liquid chromatography-mass spectrometry (LC-MS/MS). The Bicinchoninic acid assay (BCA) kit was purchased from Biodee, China. Ammonium bicarbonate, dithiothreitol (DTT), and iodoacetamide (IAA) were obtained from Sigma, USA. Acetonitrile was obtained from Thermo Fisher, USA. Spectrometry grade of trypsin was purchased from Promega, USA.

\section{Sample Collection}

The Chinese Human Milk Project (the CHMP study) recruited 1,800 participants from eight cities of Mainland China (Beijing, Guangzhou, Chengdu, Weihai, Lanzhou, Jinhua, Zhengzhou, and Harbin) to evaluate HM composition in the Chinese population. This present study used samples during 1-6 months of lactation and had been clinically registered in ClinicalTrials.gov with registration identifier NCT03675204.

The present study used cross-sectional sample by using one sample from one individual analyzed for one time. HM was collected at 9:00-11:00 in the morning from 15 to 180 days after delivery. The samples were collected from both the right and left sides of fully pumped breasts. The minimum size for each sample was $60 \mathrm{ml}$. The inclusion criteria were lactating mothers 25-35 years old, breast-fed infants 15-180 days old, physically healthy, non-smoking and non-alcoholic consumers, given birth to physically healthy infants, and signed informed consent forms. The detailed number of the samples is figured in Table 1. The
TABLE 1 | Number of samples obtained from the hospitals in eight cities in China.

\begin{tabular}{llllllll}
\hline Cities & Total samples & \multicolumn{5}{c}{ Number of samples in month } \\
\cline { 3 - 8 } & & 1 & 2 & 3 & 4 & 5 & 6 \\
\hline Beijing & 30 & 1 & 9 & 4 & 8 & 5 & 3 \\
Guangzhou & 24 & 3 & 4 & 5 & 4 & 4 & 4 \\
Chengdu & 24 & 4 & 4 & 5 & 3 & 5 & 3 \\
Weihai & 16 & 2 & 2 & 4 & 3 & 2 & 3 \\
Lanzhou & 31 & 3 & 5 & 9 & 5 & 9 & 0 \\
Jinhua & 34 & 5 & 5 & 5 & 5 & 5 & 5 \\
Zhengzhou & 30 & 3 & 7 & 5 & 5 & 5 & 5 \\
Harbin & 18 & 3 & 3 & 3 & 3 & 3 & 3 \\
\hline
\end{tabular}

obtained samples were frozen and transported to the lab and stored at a temperature of $-80^{\circ} \mathrm{C}$ until further analysis.

\section{Protein Digestion}

The procedure of protein digestion was described as previously (15). In brief, the HM samples were centrifuged at 3,000 rpm at $10^{\circ} \mathrm{C}$ for $30 \mathrm{~min}$. The fat layer was removed and the remaining parts of protein concentration were measured using the BCA kit. Based on the results of the BCA, $10 \mu \mathrm{l}$ proteins $(1 \mu \mathrm{g} / \mu \mathrm{l})$ was diluted with $100 \mu l 0.05 \mathrm{M}$ ammonium bicarbonate in $0.5 \mathrm{ml}$ Eppendorf tube. Approximately, $10 \mu \mathrm{l} 0.1 \mathrm{M}$ DTT was added. The mixture was kept in $56^{\circ} \mathrm{C}$ water-bath for $30 \mathrm{~min}$, followed by adding $15 \mu l 0.5 \mathrm{M}$ Iodoacetamide (IAA). The mixture was incubated at room temperature for $30 \mathrm{~min}$ in the dark. The protein digestion was performed by adding a mass ratio of 1:100 trypsin/protein and mildly shook at room temperature overnight. The digestion was stopped by adding 1\% formic acid. Before running in LC-MS, the samples were desalted using C-18 column.

\section{Liquid Chromatography-Mass Spectrometry}

The proteomics was performed using EASY-nLC 1200 coupled with Q Exactive HF. Generally, the samples were separated using a C18 analytical column (150 $\mu \mathrm{m}$ inner-diameter, outer-diameter $15 \mathrm{~cm}-1.9 \mu \mathrm{m}, 120 \AA$ pore size, ReproSil-Pur C18-AQ). The mobile phase was constituted by $0.1 \%$ formic acid in water and $0.1 \%$ formic acid as solution $\mathrm{A}$; and $19.9 \%$ water mixed with $80 \%$ acetonitrile as solution $\mathrm{B}$. The flow rate was $0.6 \mu \mathrm{l} / \mathrm{min}$, the column temperature was $50^{\circ} \mathrm{C}$, the gradient was $4-7 \%$ solution $\mathrm{B}$ at the initial $1 \mathrm{~min}, 7-13 \%$ solution $\mathrm{B}$ for $1-7 \mathrm{~min}, 13-25 \%$ solution B for 7-47 min, 25-40\% solution B for 47-68 min, 40955 solution B for $68-69 \mathrm{~min}$, and keeping 95\% solution B for 69-75 min. The MS setting was $2.1 \mathrm{kV}$ spray voltage. MS data were acquired by using data-dependent acquisition mode which dynamically chose the top-30 most abundant precursor ions from survey scan $(300-1,400 \mathrm{~m} / z)$ for high energy collisional dissociation (HCD) fragmentation with a resolution of 120,000 $(200 \mathrm{~m} / z)$. The MS/MS spectra were acquired in the HF normal scan mode. 


\section{Data Analysis}

The results from LC-MS/MS raw files were analyzed by using Maxquant 1.6.3.4 $(9,16)$. The Homo sapiens proteome database was downloaded from Uniprot (https://www.uniprot.org) and configured by Maxquant. In addition, the contamination database of Maxquant was adopted.

The carbamide-methylation of cysteine was set as fixed modification, and oxidation of methionine, N-terminal acetylation, and deamidation of asparagine or glutamine was set as variable modifications. Mass tolerance was set as 20 ppm for MS peaks and 0.5 Da for MS/MS peaks. The false discovery rates (FDR) was set as $1 \%$ and at least 1 peptide was required for identification. Label-free quantification (LFQ) and intensity-based absolute quantification (iBAQ) values were selected for relative protein quantification across all the samples and comparing the levels of different proteins from the same sample, respectively.

\section{Statistical Analysis}

The significant differences were analyzed using SPSS 22 (IBM, USA), and the test used was one-way ANOVA with post-hoc Tukey's HSD $(P<0.05)$. Protein differentiation and principal component analysis (PCA) were performed by XLSTAT 2021 with the Benjamini-Hochberg test $(P<0.05)$.

\section{Cluster and Gene Ontology Enrichment Analysis}

The GO enrichment of protein was performed by using DAVID Bioinformatics resources 6.8 (https://david.ncifcrf.gov) $(9,17)$. The protein-protein interaction was figured out by using STRING 9 (18).

\section{RESULTS}

\section{Identified Proteins in Eight Cities}

In the present study, 860, 760, 1,426, 1,298, 1,029, 1,029, 1,022 , and 960 proteins were identified in Beijing, Guangzhou, Chengdu, Weihai, Lanzhou, Jinhua, Zhengzhou, and Harbin, respectively. The list of identified proteins can be seen in Supplementary Table 1.

Major GO biological processes from the overall identified protein (Figure 1) showed that translational initiation had the dominant function in HM proteomes with a $p$-value of $4.52 \times 10^{-86}$. It was followed by viral transcription, cell-cell adhesion, Fc-epsilon signaling pathway, translation, complement activation, and receptor-mediated endocytosis. The metabolism function was represented by terms of proteolysis and carbohydrate metabolic process. Several immune related functions showed to be significant in the GOBP enrichment, such as antigen processing and presentation, immune response, and innate immune response with a $p$-value of $1.28 \times 10^{-28}, 4.42 \times$ $10^{-11}$, and $1.40 \times 10^{-7}$, respectively.

\section{Protein Variation Across the Cities and Lactation Periods}

The PCA showed from principal component 1 (PC1) and principal component 2 (PC2) could explain $99.37 \%$ of intensitybased protein variance among eight cities. From PC1 and PC2, the first pool consisted of Weihai, Chengdu, and Jinhua, which could be discriminated from the second pool that consisted of Zhengzhou, Beijing, Guangzhou, Lanzhou, and Harbin (Figure 2A). Those discrimination were based on several protein including LALBA, CSN2, and IGKC that were higher in the second pool. Meanwhile, CSNS1, CSN3, LTF, CEL, IGHA1, CLU, B2M, LYZ, and XDH were abundant in the first pool (Figure 2B).

Proteins among different lactation periods were figured out by using PC1 and PC2 that could explain 99.95\% of intensitybased protein variation (Figure 3). The first pool consisted of the 1st, 2nd, and 5th months of the lactation period, while the other groups consisted of the 3rd, 4th, and 5th months of the lactation period (Figure 3A). These groups were discriminated by several proteins, including LALBA, CSN1S1, CEL, LTF, B2M, SPP1, and IGHA1 that were abundant in the first pool. The second pool had a higher abundance of CSN2, CSN3, LYZ, IGKC, CLU, and PIGR (Figure 3B). The seven major proteins in all the cities were LALBA, CSN2, CSN1S1, CSN3, LTF, ALB, and CEL. The average intensity-based absolute quantification (iBAQ) value and the putative function of each protein based on the DAVID Bioinformatics were available in Table 2. Among the identified proteins, LALBA shared the highest percentage than the rest of the proteins. Zhengzhou had the highest LALBA concentration. LALBA and CSN2 in Weihai were below the other provinces on average, while the kappa-casein was the highest. Bile saltstimulated lipase (CEL), as the seventh major protein in HM across the provinces, was noticed as the highest in Jinhua.

The intensity of 184 proteins was significantly different among the cities (Supplementary Table 2), including several abundant proteins: CSN1S1, CSN2, CSN3, LTF, CEL, IGKC, IGHA1, $\mathrm{B} 2 \mathrm{M}$, and PIGR. Four proteins were significantly different during 6 months of lactation, namely CTSC, LYZ, CA6, and CHRDL2 (Supplementary Table 3). As the lactation prolonged, the intensity of CTSC, CA6, and CHRDL2 decreased, while the intensity of LYZ increased.

\section{Gene Ontology Enrichment of Significantly Different Proteins}

Gene ontology biological process (GOBP), cellular (GOCC), and Kyoto Encyclopedia of Genes and Genomes (KEGG) pathways were enriched from the significantly different proteins across the cities (Figure 4). Platelet degranulation was the most noticeable GOBP observed that had a $p$-value of $3.66 \times 10^{-27}$, and it was followed by innate immune response and triglyceride metabolic process with a $p$-value of $1.87 \times 10^{-5}$ and $5.38 \times 10^{-4}$, respectively. The white bar showed the extracellular exosome as the major GOCC, and the KEGG pathway was dominated by complement and coagulation cascades, fat digestion and 


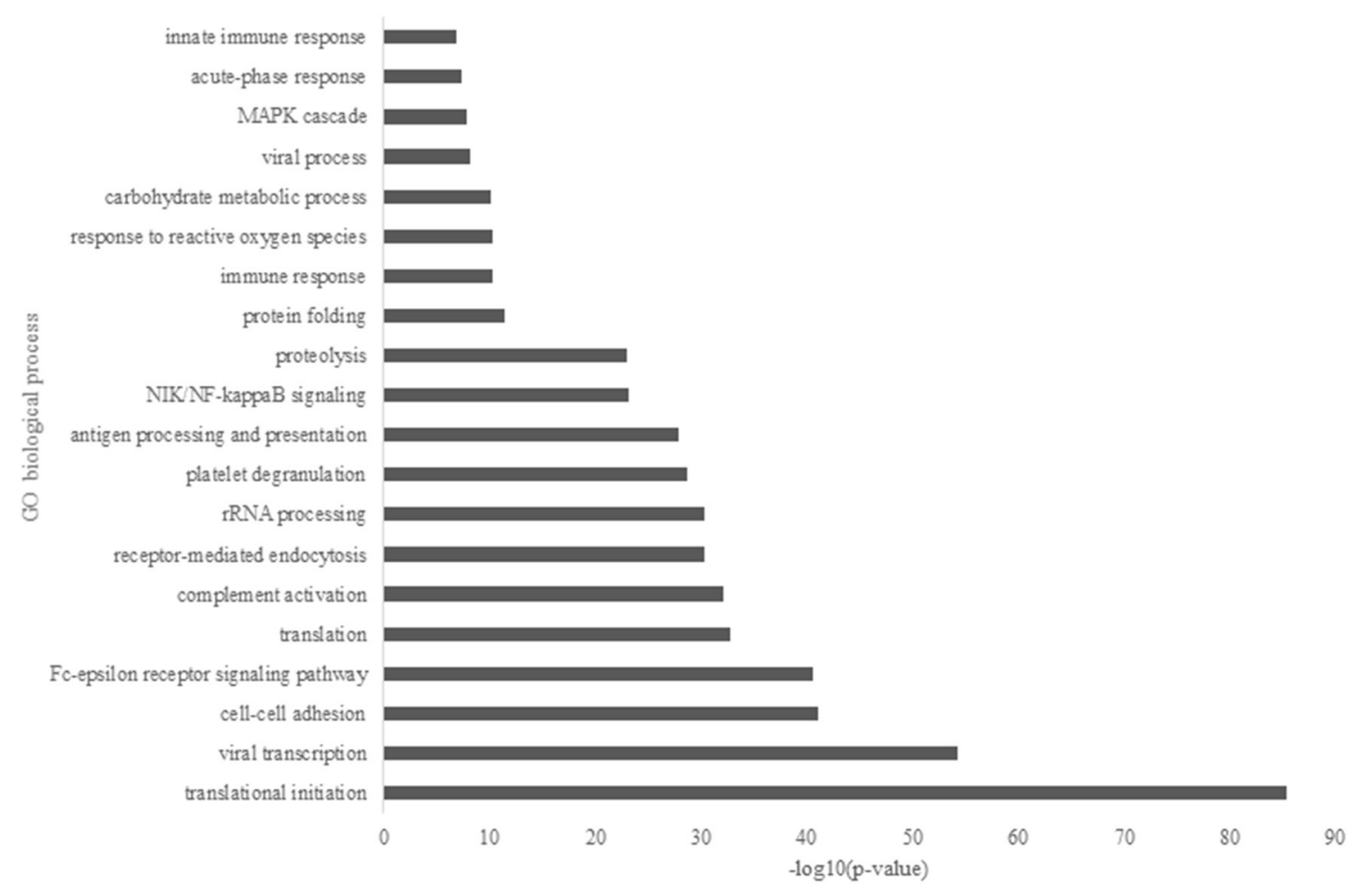

FIGURE 1 | Major gene ontology (GO) biological process of overall identified proteins in Chinese human milk (HM) enriched by the DAVID bioinformatics.
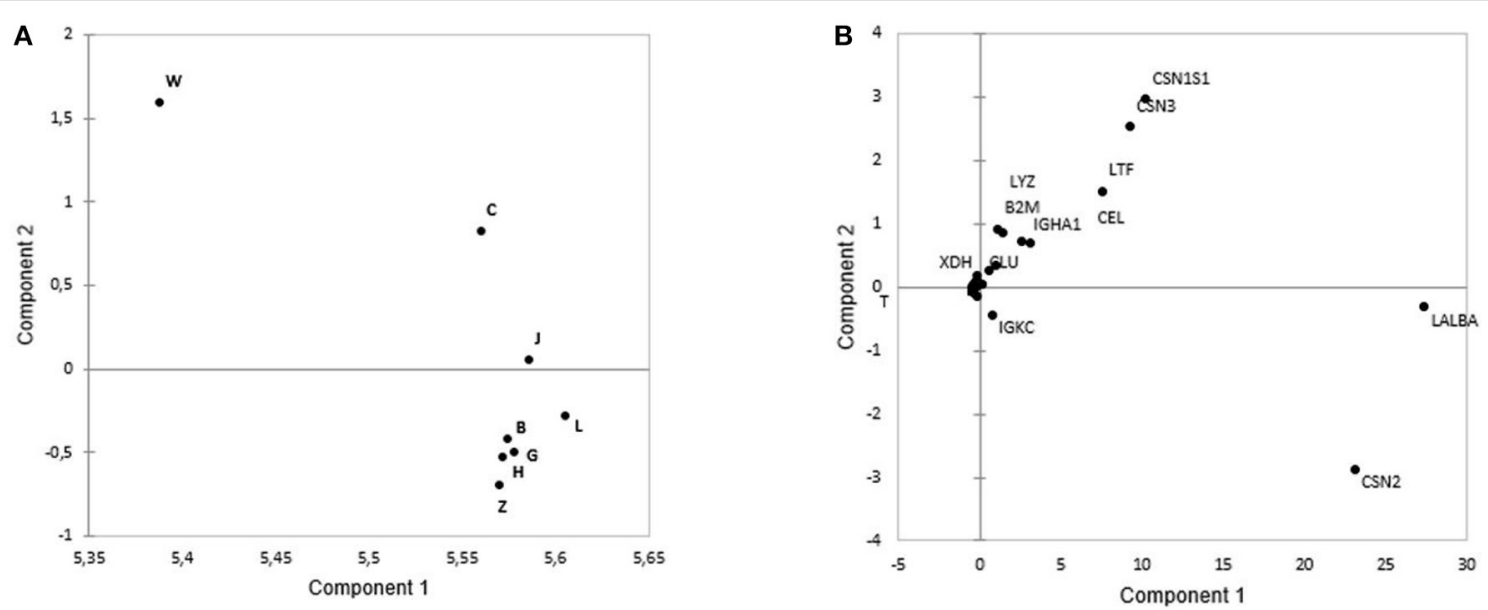

FIGURE 2 | The principal component analysis (PCA) plots based on protein intensity of: (A) B, Beijing; G, Guangzhou; C, Chengdu;W, Weihai; L, Lanzhou; J, Jinhua; Z, Zhengzhou, and H, Harbin. Weihai, Chengdu, and Jinhua are located in the same pool, while Lanzhou, Beijing, Guangzhou, Harbin, and Zhengzhou are located in the second pool. (B) Loading plots based on the intensity of the proteins showed that CSN2, LALBA, and IGKC were the abundant proteins in the second pool, while the first pool had abundance of CSN1S1, CSN3, LTF, LYZ, B2M, IGHA1, CEL, CLU, B2M, and XDH.

absorption, and peroxisome proliferator-activated receptors (PPAR) signaling pathway.

The intensity of proteins enriched in platelet degranulation, triglyceride metabolic process, and innate immune response is summed in Figure 5. Figure 5A shows the obvious different intensities in platelet degranulation across eight cities except in the 1 st month of lactation which ranged from 5 to $11 \%$ of the iBAQ values. Weihai had the highest iBAQ values in the term of platelet degranulation during the 1st to 5th months of the lactation period. Meanwhile, in the 6th month Weihai and Chengdu were not significantly different based on the HSD posthoc test. Zhengzhou consistently had the lowest intensities during 

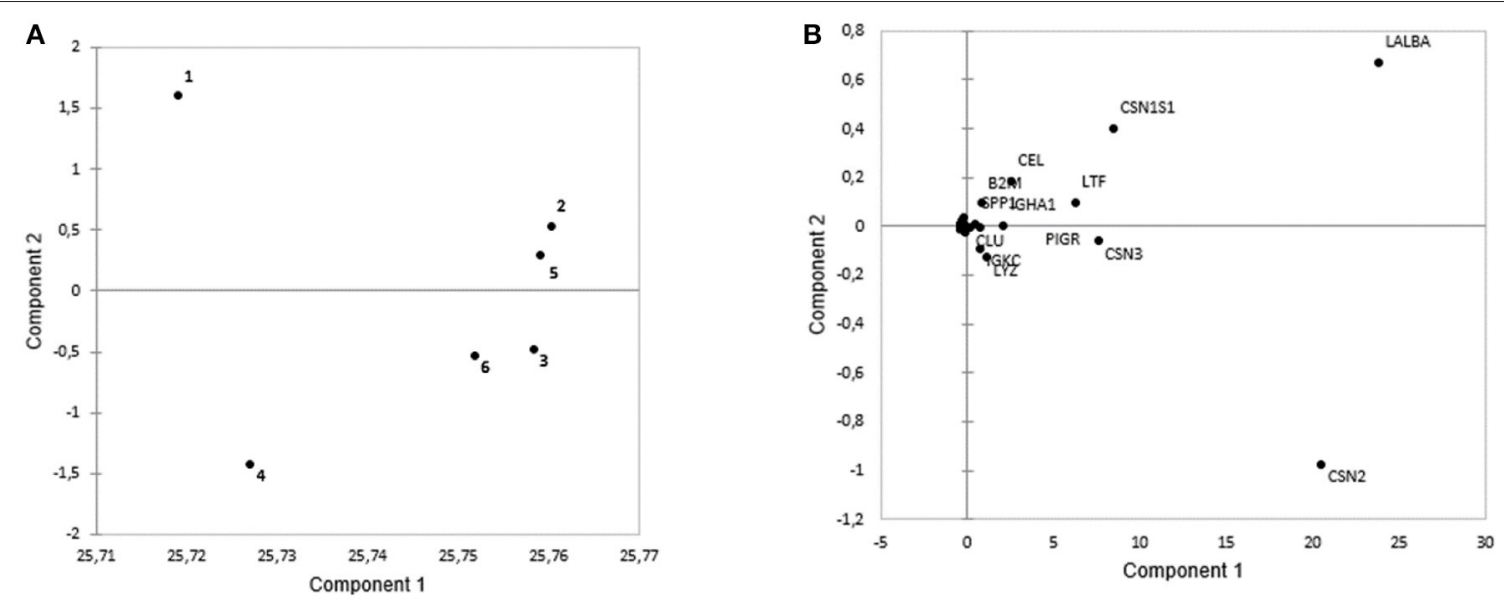

FIGURE 3 | The PCA plots based on protein intensity of: (A) Different lactation periods during the 6 months of lactation. The first pool includes the 1st, 2nd, and 5th month of lactation, while second pool includes the 3rd, 6th, and 4th month of lactation. (B) Loading plots based on intensity of the proteins based on the different lactation periods. The first pool was abundant in LALBA, CSN1S1, CEL, LTF, B2M, SPP1, and IGHA1. Meanwhile, the second pool was abundant with CLU, PIGR, IGKC, LYZ, and CSN.

TABLE 2 | Most abundant protein identified in eight cities.

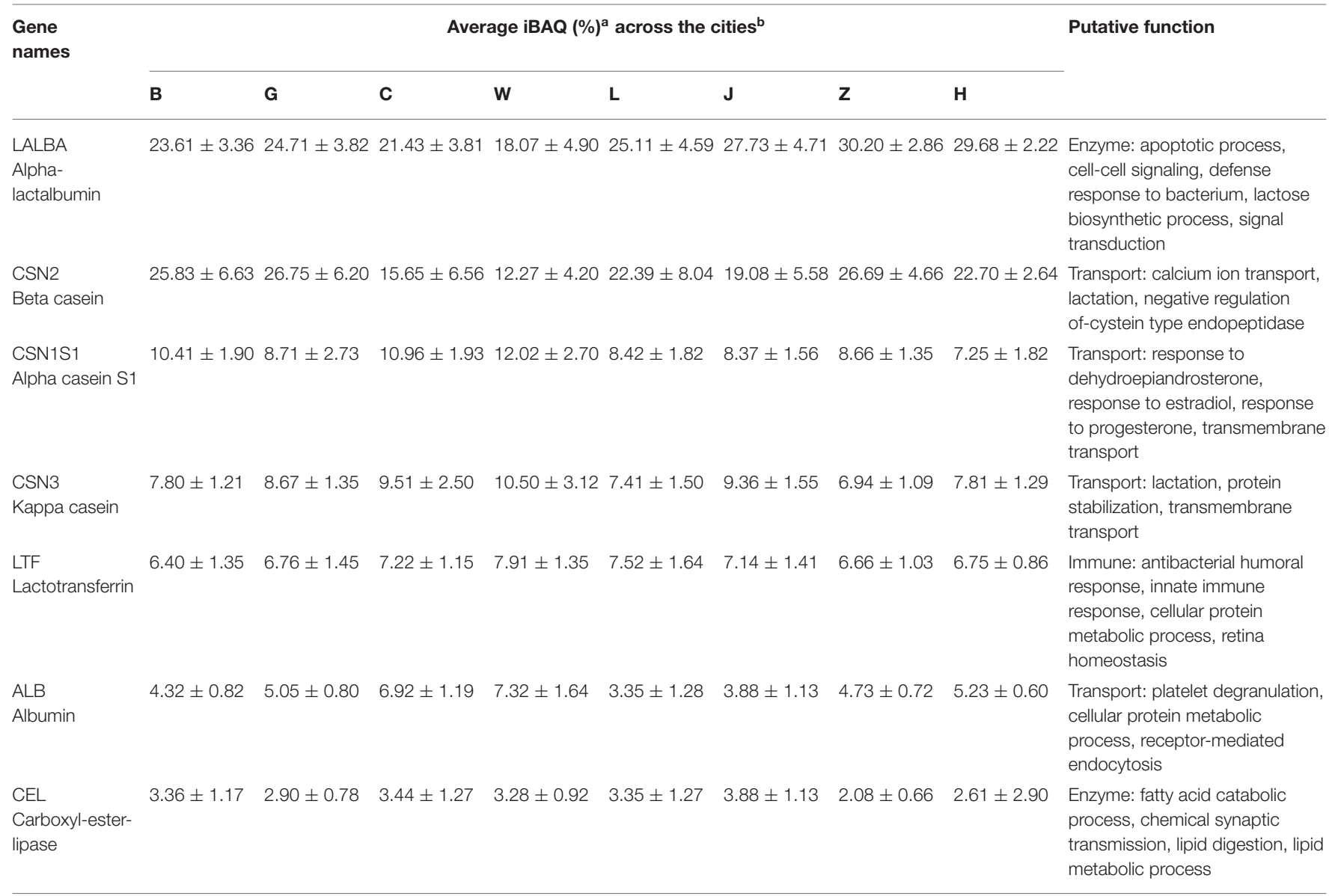

${ }^{a}$ Accounted as average of iBAQ during 6 months of lactation.

${ }^{b}$ B, Beijing; G, Guangzhou; C, Chengdu; W, Weihai; L, Lanzhou; J, Jinhua; Z, Zhengzhou; H, Harbin. 


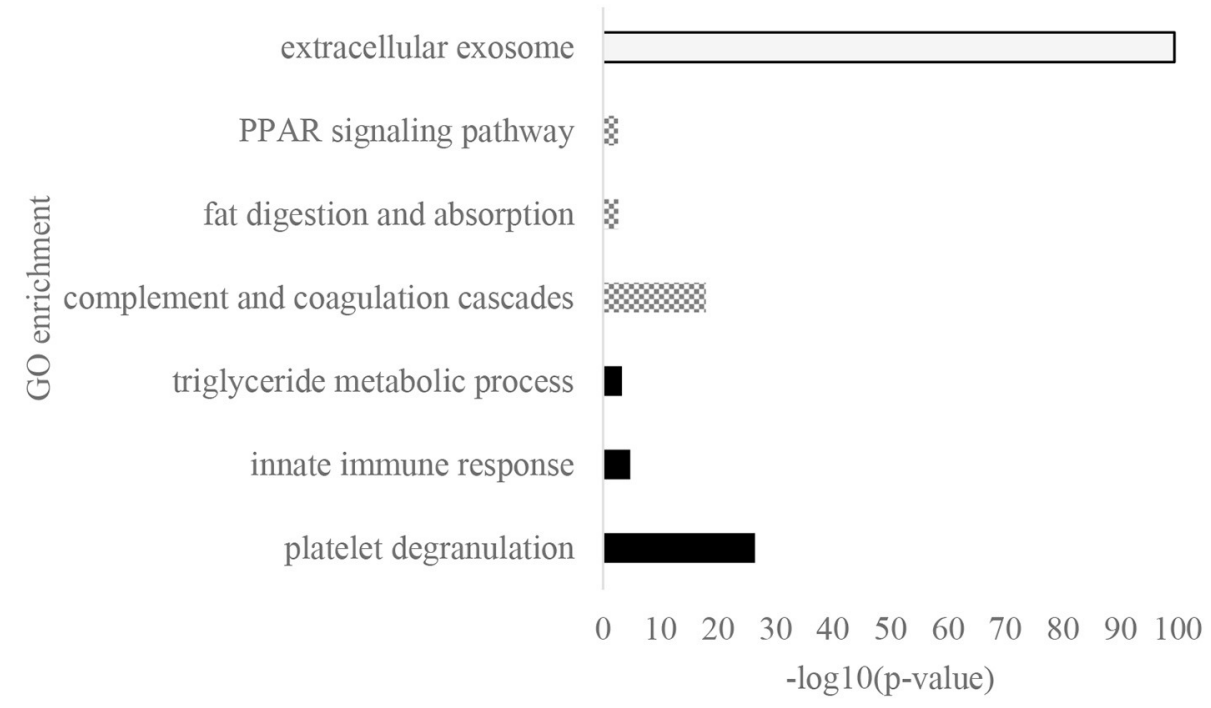

FIGURE 4 | The GO enrichment of significantly different protein across eight cities. White: GO cellular location, dominated by extracellular exosome; Gray: Kyoto Encyclopedia of Genes and Genomes (KEGG) pathways that consisted of complement coagulation cascades, fat digestion and absorption, and Peroxisome proliferator-activated receptors (PPAR) signaling pathway; and Black: GO biological process, dominated by platelet degranulation, innate immune response, and triglyceride metabolic process.

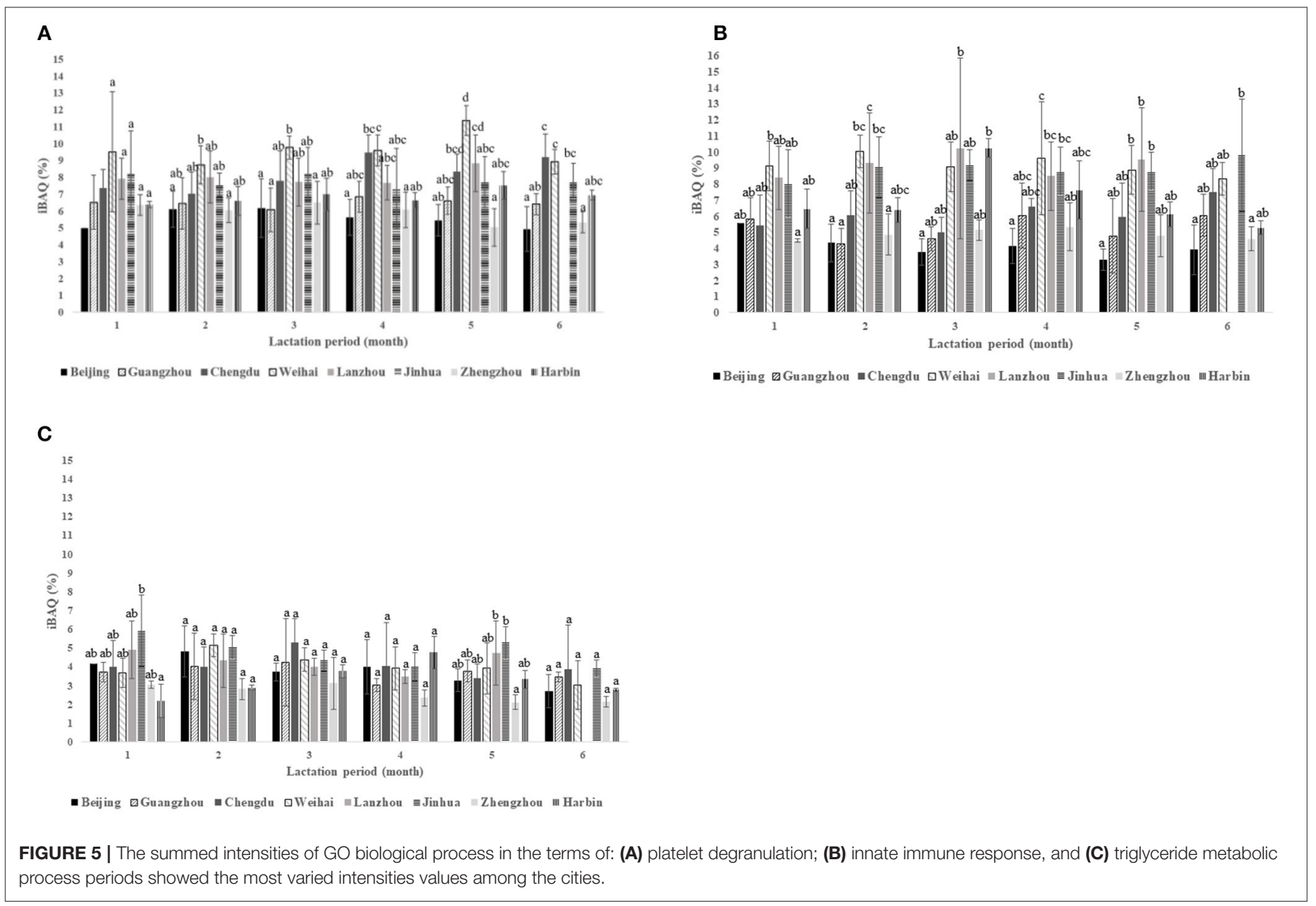




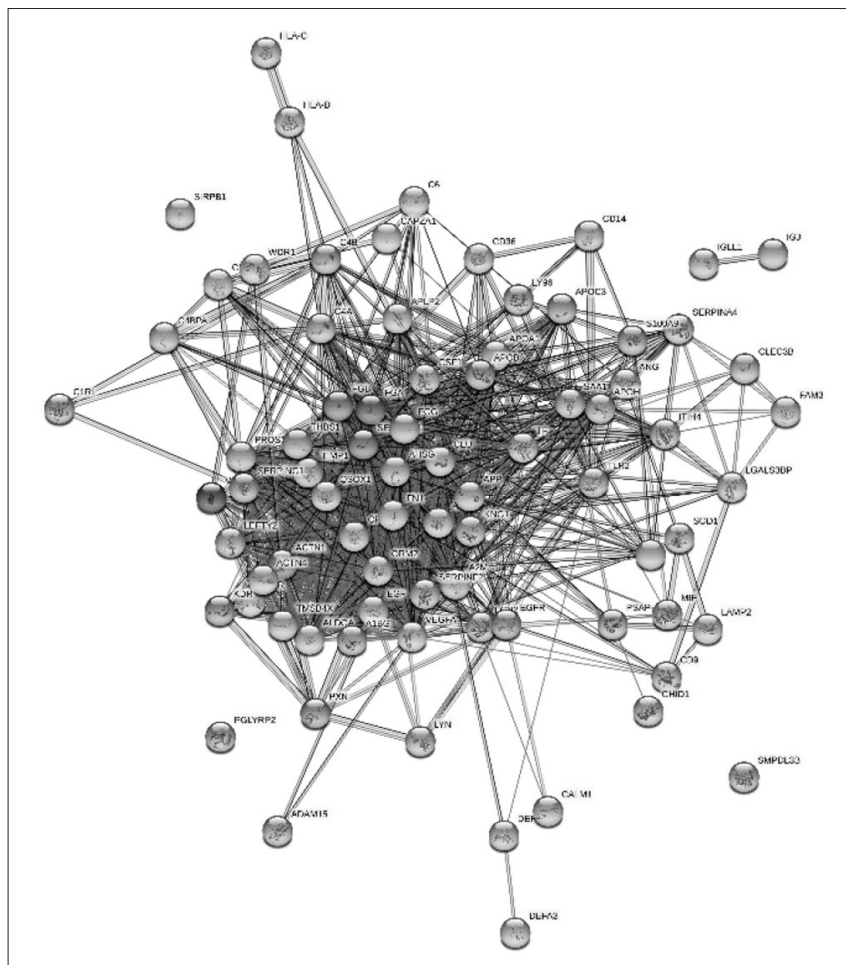

FIGURE 6 | Interaction of platelet degranulation and innate immune response protein in Chinese HM were enriched with DAVID Bioinformatics and visualized using STRING 9.

the periods, while Harbin also shared the insignificant difference with Zhengzhou except in the 5th month of lactation.

Remarkable different protein intensities were found during 6 months of the lactation period in terms of innate immune response that ranged from 4 to $11 \%$ of the iBAQ values (Figure 5B), especially in Weihai, Lanzhou, and Jinhua, which had higher intensities among the other cities. In contrast, Beijing and Zhengzhou were lower in BBAQ values compared with the others. In this term, the significant variation was observed along the lactation period in each city. The 2 nd and 4 th months had more variation intensities compared to that of the other periods.

The triglyceride metabolic process, which ranged from 2 to $6 \%$ of the iBAQ values, revealed a significant difference in the 1st and 5 th months of the lactation period (Figure 5C). Meanwhile, no significant difference was observed during the rest of the lactation period. Harbin had the lowest intensities in the 1st month of the lactation period; in contrast, Jinhua was the highest. However, in the 5th month, Zhengzhou had the lowest iBAQ values, and Jinhua still had the highest.

The proteins from platelet degranulation and innate immune response were illustrated by STRING 9 to see the proteinprotein interactions since both the terms were enriched under the complement and coagulation cascade in the KEGG pathways (Figure 6). Almost all of the proteins were connected except the SMPDL28, SIRPB1, IGLL, and IGJ.

\section{DISCUSSIONS}

\section{Human Milk Proteome Varied Across the Cities and Lactation Periods}

Human milk composition is suited for infant needs and growth. However, the profile of HM is crucial for developing products for infants who either cannot access their mother's milk or whose mothers' milk production volume was insufficient. The HM variation in the different continents had been reported previously using the metabolomics method (11). The present method found a higher number of identified proteins than the previous study (13). It means our proteomics method was comparable to the other methods and could be deeper in the bioinformatics enrichment since more proteins were identified to interpret its biological function.

Twelve proteins were the discriminants of the cities, namely LALBA, CSN2, IGKC, CSN1S1, CSN3, LTF, CEL, IGHA1, B2M, $\mathrm{LYZ}$, CLU, and XDH. These discriminant proteins were mainly contributed as host defense in the biological process of an infant. It was agreed with a previous research that found the immune protein including lactalbumin, lactoferrin, and IgA were varied among the population as a function of pathogen pressure of the environment (19).

In the most abundant protein in HM, this study found that CSN1S1, CSN2, and CSN3, which belonged to casein, were statistically different across the cities. Casein plays the role of primary source of phosphate and calcium in HM due to its function in casein-micelle aggregates in the calcium transport process $(20,21)$. This finding was in line with a prior study that reported the remarkable difference of kappa and beta casein in HM serum of Chinese and Dutch mothers (14). The LTF variations across the cities were in line with previous findings in Chinese HM across distinct regions (22).

Based on differential expression tests, variation of significant proteins across the cities was very high that presented in Supplementary Table 2 . A total of 184 proteins were significantly different across the city with a $p$-value of below 0.05 that was followed by enrichment in Figure 4. Meanwhile, only four proteins were statistically different during the 6 months of lactation, namely CTSC, LYZ, CA6, and CHRDL2. LYZ was upregulated while CTSC, CA6, and CHRDL2 were downregulated. The downregulated CHRDL2 was in line with a prior proteomics research in HM over lactation (3). Since the function of CHRDL2 is related to ossification, it might be possible that the expression was downregulated by organ maturation throughout the 6 months. The increase of LYZ in the human mature milk was previously described by Montagne et al. as the passive protective agents of breast-fed infants during mature lactation (23).

In Figure 2, the first pool consisted of the 1st, 2nd, and 5th months of the lactation period that had a high abundance of LALBA, CSN1S1, CEL, LTF, B2M, SPP1, and IGHA1. The high abundance of LALBA and CSN1S1 in the beginning months of the lactation had been previously reported in bovine milk (24). B2M, IGHA1, and LTF as the proteins related to antibacterial humoral response were logical to have a high abundance in the 1st and 2nd months of lactation since newborns had 
little protection against bacteria from the environment. CEL, as the main protein related to fat metabolism, was reported to be highly abundant during the initial months (3) since it functions as an immature pancreatic lipase substitution. Meanwhile, the highly percentage of aforementioned proteins in the fifth month needs to be further investigated. We speculated it was because of the highly individual variation in the 5th month samples.

\section{Significant Different Proteins Across the Cities Were Linked to Newborn Protection}

The significantly different proteins across the cities were involved in platelet degranulation, and those proteins were mainly located in the extracellular exosome (Figure 4). It was agreed with a previous finding in proteomics of bovine milk exosome that showed platelet degranulation was the most significant biological process (25). Beyond its primary role in thrombus or plug formation in wound healing, the platelet also worked in tissue repair, angiogenesis, inflammation, and host defense (26-30). In newborns, overall platelet degranulation released during platelet activation was lower compared to adults (31). Based on our results, there was no significant difference in platelet degranulation in the 1st month across the cities (Figure 5A). It might indicate that the newborns demanded HM proteins to regulate platelet degranulation for completing platelet function since platelets activation was low in the first few days of life (32).

Neonates were generally believed to have partially immunological incompetence and were susceptible to infections. Platelets also played a role in the host defense of the newborns. By the present result, platelet degranulation shared similar proteins with the innate immune response in GOBP, including FGA, FGB, CLU, SERPING1, and CD36. All proteins contributed in both terms were figured out in protein network connection (Figure 6). Platelet glycoprotein 4 (CD36) had a responsibility in the phagocytosis process (31). Phagocytosis was an important innate immune response process to protect infants from microbes. CLU is a highly glycosylated protein that was linked to cell damage and apoptosis. This protein was found to be overexpressed at stressed tissues to provide a chaperone-like activity and prevent other proteins from damage (33). FGA is known as the main protein in handling fibrin production as one of the primary components of blood clots. It also acts as a fibrin deposition process that is associated with infection, where it protects against IFNG-mediated hemorrhage. The link of platelet degranulation and immune response as the host defense were regulated in complement and coagulation cascades, which were enriched in major KEGG pathway (Figure 4).

Immune response plays important roles in infants, especially innate immune response in newborns since adaptive immune response is not well-developed yet. The function of HM as a complementary immune system for newborns has been previously reported (34). From our identified protein (Supplementary Table 1), above 54 proteins were enriched as innate immune response in biological process with $p$-value
$1.6 \times 10^{-10}$. It was higher than previous findings that used a similar GO enrichment tool in HM (8). That research found the $p$-value of the immune system to be $1.8 \times 10^{-7}$. The reason might be because this study had higher identified proteins. Based on the significant expressed protein enrichment, the $p$-value of the innate immune response was $1.87 \times 10^{-5}$, which consisted of 17 proteins, namely FGB, FGA, IGHM, CFI, CLU, IGHG4, C4A, IGHG1, IGHG2, IGKC, IGLL1, SERPING1, CD14, IGHA1, SMPDL3B, IGHA2, and B2M. These 17 proteins brought variation to the quantitative amount of innate immune response across the cities (Figure 5B) that showed significant variations in each period of the lactation. The differences of immune-related protein intensity were also previously found in the different geographic location and ethnicity in China because the environment could influence the pathogen (13). However, other factors such as the health condition or infection of the infant should be further observed, since the infant infection could upregulated immune proteins in the HM, especially in the first year of lactation $(35,36)$.

\section{Triglyceride Metabolism Variations Across the Cities}

Breastfeed infants have better lipid utilization compared to formula-feed infants (37-39). Since delivery, infants used fat as the major energy source that contributed about $40-55 \%$ of the total energy and could obtain $5.5 \mathrm{~kg}$ fat intake during 6 months of lactation $(40,41)$. Triglycerides represented 98-99\% of total fat in HM (42). Fat metabolism and fat absorption were noticed as the enriched KEEG pathway from the significantly expressed proteins (Figure 4). The crucial protein that handled fat metabolism in HM was CEL, which was found to be the seventh abundant protein (Table 2 ) and also significantly expressed protein across the cities (Supplementary Table 2). CEL from HM played a major role in infant lipid utilization by replacing pancreatic triglycerides lipase (PTL), which is secreted by the pancreas, while the pancreas condition is immature $(38,43)$. The present research found five significantly expressed proteins in different cities, namely CEL, APOH, APOA2, LPL, and APOE which were enriched in the triglycerides metabolic process. These proteins brought remarkable variation in the triglyceride metabolic process (Figure 5C), particularly in the 1 st and 5th months of the lactation period. This could be noteworthy because the lower abundance of the triglyceride metabolic process proteins might lead to lower weight gain of the infant since fat should be the major energy intake. In addition, the immature digestion tract of the infant needs HM protein to aid fat digestion (43).

However, bigger samples in a longitudinal study during the lactation stages will give more benefit to fill the gaps in this research as well as decrease the variations among individual mothers. It will bring a deeper quality of biological role interpretation as the basic data for developing the most suitable infant formula for babies who cannot obtain HM in the exclusive breastfeeding period. 


\section{CONCLUSION}

This present research found a noticeable variation of HM proteins across eight cities in China and four significantly expressed proteins during the 6 months of lactation. The 184 significantly expressed proteins across the cities mainly influenced the infant biological process in terms of platelet degranulation, innate immune response, and triglyceride metabolic process. This research could be a good recommendation for developing specialized region infant formula or HM fortifiers during the exclusive breastfeeding period.

\section{DATA AVAILABILITY STATEMENT}

The raw data supporting the conclusions of this article will be made available by the authors, without undue reservation.

\section{ETHICS STATEMENT}

The studies involving human participants were reviewed and approved by National Library of Medicine. The patients/participants provided their written informed consent to participate in this study.

\section{REFERENCES}

1. Pham Q, Patel P, Baban B, Yu J, Bhatia J. Factors affecting the composition of expressed fresh human milk. Breastfeed Med. (2020) 15:5518. doi: 10.1089/bfm.2020.0195

2. Bhandari N, Chowdhury R. Infant and young child feeding. Proc Indian Natl Sci Acad. (2016) 82:1507-17. doi: 10.16943/ptinsa/2016/48883

3. Zhang L, de Waard M, Verheijen H, Boeren S, Hageman JA, van Hooijdonk $\mathrm{T}$, et al. Changes over lactation in breast milk serum proteins involved in the maturation of immune and digestive system of the infant. J Proteomics. (2016) 7:362-5. doi: 10.1016/j.dib.2016.02.046

4. Ballard O, Morrow AL. Human milk composition. nutrients and bioactive factors. Pediatr Clin North Am. (2013) 60:4974. doi: 10.1016/j.pcl.2012.10.002

5. Lönnerdal B. Nutritional and physiologic significance of human milk proteins. Am J Clin Nutr. (2003) 77:1537S-43S. doi: 10.1093/ajcn/77.6.1537S

6. Palmer DJ, Kelly VC, Smit AM, Kuy S, Knight CG, Cooper GJ. Human colostrum: Identification of minor proteins in the aqueous phase by proteomics. Proteomics. (2006) 6:2208-16. doi: 10.1002/pmic.200500558

7. Roncada P, Stipetic LH, Bonizzi L, Burchmore RJS, Kennedy MW. Proteomics as a tool to explore human milk in health and disease. J Proteomics. (2013) 88:47-57. doi: 10.1016/j.jprot.2013.04.008

8. Gao X, McMahon RJ, Woo JG, Davidson BS, Morrow AL, Zhang Q. Temporal changes in milk proteomes reveal developing milk functions. J Proteome Res. (2012) 11:3897-907. doi: 10.1021/pr3004002

9. Lu J, Liu L, Pang X, Zhang S, Jia Z, Ma C, et al. Comparative proteomics of milk fat globule membrane in goat colostrum and mature milk. Food Chem. (2016) 209:10-16. doi: 10.1016/j.foodchem.2016.04.020

10. Lu J, Zhang S, Liu L, Pang X, Ma C, Jiang S, et al. Comparative proteomics analysis of human and ruminant milk serum reveals variation in protection and nutrition. Food Chem. (2018) 261:274-82. doi: 10.1016/j.foodchem.2018.04.065

11. Carlos G, Morales JM, Monle D, Toit E, Kumar H, Linderborg KM, et al. Specific geographical locations and its association with the milk microbiota. Nutrients. (2018) 10:1-20. doi: 10.3390/nu10101355

\section{AUTHOR CONTRIBUTIONS}

JLu: conceptualization and validation. WZ and JLu: methodology. RS and WZ: software and investigation. WZ: formal analysis. JLu, JP, and YL: resources. RS: data curation, writing-original draft preparation, and visualization. HZ, $\mathrm{XP}, \mathrm{SZ}$, and JLu: writing-review and editing. JLu and JLv: supervision. SJ, JLu, and JLv: project administration. JP, SJ, JLu, and JLv: funding acquisition. All authors have read and agreed to the published version of the manuscript.

\section{FUNDING}

This research was supported by Bai-Qian-Wan Engineering and Technology Master Project (Grant \#2019ZX07B01, funded by the Government of Heilongjiang Province of the People's Republic of China) and Feihe's Internal CHMP study grant.

\section{SUPPLEMENTARY MATERIAL}

The Supplementary Material for this article can be found online at: https://www.frontiersin.org/articles/10.3389/fnut.2021. 682429/full\#supplementary-material

12. Kumar H, Toit E, Kulkarni A, Aakko J, Collado MC, Salminen S, et al. Distinct patterns in human milk microbiota and fatty acid profiles across specific geographic locations. Front Microbiol. (2016) 7:112. doi: 10.3389/fmicb.2016.01619

13. Zhang L, Ma Y, Yang Z, Jiang S, Liu J, Hettinga KA, et al. Geography and ethnicity related variation in the Chinese human milk serum proteome. Food Funct. (2019) 10:7818-27. doi: 10.1039/C9FO01591D

14. Elwakiel M, Boeren S, Hageman JA, Szeto IM, Schols HA, Hettinga KA. Variability of serum proteins in Chinese and dutch human milk during lactation. Nutrients. (2019) 11:1-14. doi: 10.3390/nu11030499

15. Lu J, Wang X, Zhang W, Liu L, Pang X, Zhang S, et al. Comparative proteomics of milk fat globule membrane in different species reveals variations in lactation and nutrition. Food Chem. (2016) 196:66572. doi: 10.1016/j.foodchem.2015.10.005

16. Tyanova S, Temu T, Cox J. The MaxQuant computational platform for mass spectrometry-based shotgun proteomics. Nat Protoc. (2016) 11:230119. doi: $10.1038 /$ nprot.2016.136

17. Huang DW, Sherman BT, Lempicki RA. Systematic and integrative analysis of large gene lists using DAVID bioinformatics resources. Nat Protoc. (2009) 4:44-57. doi: 10.1038/nprot.2008.211

18. Szklarczyk D, Gable AL, Lyon D, Junge A, Wyder S, Huerta-Cepas J, et al. STRING v11: protein-protein association networks with increased coverage, supporting functional discovery in genome-wide experimental datasets. Nucleic Acids Res. (2019) 47:D607-13. doi: 10.1093/nar/gky1131

19. Klein LD, Huang J, Quinn E, Martin MA, Breakey AA, Gurven M, et al. Variation among populations in the immune protein composition of mother's milk reflects subsistence pattern. Evol Med Public Heal. (2018) 2018:23045. doi: 10.1093/emph/eoy031

20. Poth AG, Deeth HC, Alewood PF, Holland JW. Analysis of the human casein phosphoproteome by 2-D electrophoresis and MALDI-TOF/TOF MS reveals new phosphoforms. J Proteome Res. (2008) 7:5017-27. doi: 10.1021/pr800387s

21. Liao Y, Weber D, Xu W, Durbin-Johnson BP, Phinney BS, Lönnerdal B. Absolute quantification of human milk caseins and the whey/casein ratio during the first year of lactation. J Proteome Res. (2017) 16:411321. doi: 10.1021/acs.jproteome.7b00486 
22. Yang Z, Jiang R, Chen Q, Wang J, Duan Y, Pang X, et al. Concentration of lactoferrin in human milk and its variation during lactation in different Chinese populations. Nutrients. (2018) 10:1-10. doi: 10.3390/nu10091 235

23. Montagne $\mathrm{P}$, Cuillière $\mathrm{ML}$, Molé $\mathrm{C}$, Béné $\mathrm{MC}$, Faure $\mathrm{G}$. Changes in lactoferrin and lysozyme levels in human milk during the first twelve weeks of lactation. Adv Exp Med Biol. (2001) 501:241-7. doi: 10.1007/978-1-4615-1371-1_30

24. Sigl T, Meyer HHD, Wiedemann S. Gene expression of six major milk proteins in primary bovine mammary epithelial cells isolated from milk during the first twenty weeks of lactation. Czech J Anim Sci. (2012) 57:46980. doi: 10.17221/6347-CJAS

25. Samuel M, Chisanga D, Liem M, Keerthikumar S, Anand S, Ang CS, et al. Bovine milk-derived exosomes from colostrum are enriched with proteins implicated in immune response and growth. Sci Rep. (2017) 7:110. doi: 10.1038/s41598-017-06288-8

26. Mcmorran BJ, Marshall VM, Graaf C De, Drysdale KE, Shabbar M, Smyth GK, et al. Survival to infection. Platelets. (2009) 323:797-800. doi: 10.1126/science.1166296

27. Italiano JE, Richardson JL, Patel-Hett S, Battinelli E, Zaslavsky A, Short S, et al. Angiogenesis is regulated by a novel mechanism: pro- and antiangiogenic proteins are organized into separate platelet $\alpha$ granules and differentially released. Blood. (2008) 111:1227-33. doi: 10.1182/blood-2007-09-113837

28. Vieira-de-Abreu A, Campbell RA, Weyrich AS, Zimmerman GA. Platelets: versatile effector cells in hemostasis, inflammation, and the immune continuum. Semin Immunopathol. (2012) 34:5-30. doi: 10.1007/s00281-011-0286-4

29. Youssefian T, Drouin A, Massé JM, Guichard J, Cramer EM. Host defense role of platelets: Engulfment of HIV and Staphylococcus aureus occurs in a specific subcellular compartment and is enhanced by platelet activation. Blood. (2002) 99:4021-29. doi: 10.1182/blood-2001-12-0191

30. Leslie M. Beyond clotting: the powers of platelets. Science. (2010) 328:5624. doi: 10.1126/science.328.5978.562

31. Esiaba I, Mousselli I, Faison GM, Angeles DM, Boskovic DS. Platelets in the newborn. Neonatal Med. (2019) 10:1-31. doi: 10.5772/intechopen.86715

32. Sitaru AG, Holzhauer S, Speer CP, Singer D, Obergfell A, Walter U, et al. Neonatal platelets from cord blood and peripheral blood. Platelets. (2005) 16:203-10. doi: 10.1080/09537100400016862

33. Charlwood J, Hanrahan S, Tyldesley R, Langridge J, Dwek M, Camilleri P. Use of proteomic methodology for the characterization of human milk fat globular membrane proteins. Anal Biochem. (2002) 301:31424. doi: 10.1006/abio.2001.5498

34. Burris AD, Pizzarello C, Järvinen KM. Immunologic components in human milk and allergic diseases with focus on food allergy. Semin Perinatol. (2021) 45:151386. doi: 10.1016/j.semperi.2020.151386

35. Li C, Liu Y, Jiang Y, Xu N, Lei J. Immunomodulatory constituents of human breast milk and immunity from bronchiolitis. Ital J Pediatr. (2017) 43:17. doi: 10.1186/s13052-017-0326-3
36. Gridneva Z, Lai CT, Rea A, Tie WJ, Ward LC, Murray K, et al. Human milk immunomodulatory proteins are related to development of infant body composition during the first year of lactation. Pediatr Res. (2020) 89:113. doi: 10.1038/s41390-020-0961-z

37. Hamosh M. Lipid metabolism in pediatric nutrition. Pediatr Clin North Am. (1995) 42:839-59. doi: 10.1016/S0031-3955(16)39020-4

38. He X, McClorry S, Hernell O, Lönnerdal B, Slupsky CM. Digestion of human milk fat in healthy infants. Nutr Res. (2020) 83:15-29. doi: 10.1016/j.nutres.2020.08.002

39. Nguyen TTP, Bhandari B, Cichero J, Prakash S. In vitro lipolysis of dairy and soy based infant formula. Food Res Int. (2018) 106:696705. doi: 10.1016/j.foodres.2018.01.003

40. Zou X, Ali AH, Abed SM, Guo Z. Current knowledge of lipids in human milk and recent innovations in infant formulas. Curr Opin Food Sci. (2017) 16:28-39. doi: 10.1016/j.cofs.2017.06.010

41. Koletzko B, Agostoni C, Bergmann R, Ritzenthaler K, Shamir R. Physiological aspects of human milk lipids and implications for infant feeding: a workshop report. Acta Paediatr Int J Paediatr. (2011) 100:140515. doi: 10.1111/j.1651-2227.2011.02343.x

42. Mazzocchi A, D’Oria V, De Cosmi V, Bettocchi S, Milani GP, Silano M, et al. The role of lipids in human milk and infant formulae. Nutrients. (2018) 10:1-14. doi: 10.3390/nu10050567

43. Lindquist $S$, Hernell $O$. Lipid digestion and absorption in early life : an update. Curr Opin Clin Nutr Metab Care. (2010) 13:314-20. doi: 10.1097/MCO.0b013e328337bbf0

Conflict of Interest: JP, YL, and SJ were employed by the company Heilongjiang Feihe Dairy Co., Ltd.

The remaining authors declare that the research was conducted in the absence of any commercial or financial relationships that could be construed as a potential conflict of interest.

Publisher's Note: All claims expressed in this article are solely those of the authors and do not necessarily represent those of their affiliated organizations, or those of the publisher, the editors and the reviewers. Any product that may be evaluated in this article, or claim that may be made by its manufacturer, is not guaranteed or endorsed by the publisher.

Copyright (c) 2021 Sari, Pan, Zhang, Li, Zhu, Pang, Zhang, Jiang, Lu and Lv. This is an open-access article distributed under the terms of the Creative Commons Attribution License (CC BY). The use, distribution or reproduction in other forums is permitted, provided the original author(s) and the copyright owner(s) are credited and that the original publication in this journal is cited, in accordance with accepted academic practice. No use, distribution or reproduction is permitted which does not comply with these terms. 